\title{
Técnica Educativa para Mejorar los Conocimientos de Salud Oral de Madres y Reducir el Índice de Higiene Oral de Preescolares
}

\author{
Educational Technique to Improve the Knowledge of Oral Health \\ of Mothers and Reduce the Index of Oral Hygiene of Preschoolers
}

Welser Emilio Muñoz-Cabrera* \& Manuel Antonio Mattos-Vela*

MUÑOZ-CABRERA, W. E. \& MATTOS-VELA, M. A. Técnica educativa para mejorar los conocimientos de salud oral de madres y reducir el índice de higiene oral de preescolares. Int. J. Odontostomat., 9(2):321-327, 2015.

RESUMEN: El objetivo de este estudio fue determinar la eficacia de la técnica educativa Desarrollo de Habilidades para mejorar los conocimientos de salud oral de madres y reducir el índice de higiene oral de preescolares. Materiales y Métodos: se hizo un estudio cuasi-experimental, se aplicó un cuestionario validado sobre conocimientos de salud oral a 84 madres antes y después de la aplicación de las técnicas educativas Desarrollo de Habilidades y Expositiva (grupo control). Las madres fueron asignadas al grupo experimental y control en forma aleatoria. Se evaluó la higiene oral de sus hijos preescolares al inicio, a los 30 días y a los 60 días, utilizando el índice simplificado de Greene-Vermillion. Al inicio la media del nivel de conocimientos de salud oral fue de 6,57 para el grupo experimental y de 6,55 para el grupo control. Después de la intervención la media del grupo experimental fue de 11,88 y del grupo control 8,48, siendo esta diferencia estadísticamente significativa $(p<0,001)$. En lo que se refiere a la higiene oral, las medias del índice al inicio, 30 y 60 días fueron en el grupo experimental 2,$51 ; 1,14$ y 0,73 mientras que para el grupo control fueron 2,$51 ; 2,02$ y 1,69 respectivamente. Hubo diferencias estadísticamente significativas entre ambos grupos a los 30 y 60 días $(p<0,001)$. La técnica Desarrollo de Habilidades demostró ser más eficaz en el aumento del nivel de conocimientos de salud oral de madres y en la disminución del índice de higiene oral de preescolares.

PALABRAS CLAVE: conocimiento, madres, salud oral, índice de higiene oral.

\section{INTRODUCCIÓN}

Las enfermedades de la cavidad oral, además de los determinantes socio-ambientales, están muy relacionadas con la higiene oral y deben ser consideradas como importantes problemas de salud pública debido a su prevalencia e incidencia en todo el mundo (Petersen, 2003).

La placa dentobacteriana es uno de los principales factores responsables de la caries dental, sin embargo, puede ser eliminada a través de una correcta higiene oral que incluya un cepillado frecuente, disminuyendo así el riesgo de presentar esta enfermedad oral (Medina et al., 2006). Diversos autores han demostrado que la mayoría de pacientes que presentan mala higiene oral tienen altos porcentajes de ca- ries dental (Castañeda et al., 2009), y que existe asociación de hábitos de higiene oral con la presencia de caries dental (Granville-García et al., 2010; Zaror et al., 2011).

Según el último estudio nacional en Perú, la prevalencia de caries en escolares de 12 años de edad provenientes de instituciones educativas estatales fue de $86,6 \%$ con un índice de CPOD de 3,67, valor elevado entre los países de América Latina (Ministerio de Salud del Perú, 2005).

Para disminuir el riesgo de desarrollar caries de infancia temprana la Academia Americana de Odontología Pediátrica promueve medidas preventivas pro-

\footnotetext{
" Doctor en Salud Pública, Dirección Regional de Salud del Callao, Callao, Perú.

"* Magíster en Estomatología, Docente de la Facultad de Odontología, Universidad Nacional Mayor de San Marcos, Lima, Perú.
} 
fesionales y en casa, que incluyen: implementar medidas de higiene oral a partir de la erupción del primer diente primario. El cepillado debe ser realizado por sus padres dos veces al día, utilizando un cepillo de dientes suave de tamaño adecuado a la edad (American Academy of Pediatric Dentistry, 2011-2012). La disminución de caries dental se da como resultado del inicio temprano de medidas educativo-preventivas destinadas y aplicadas a padres de familia (Elías, 2003).

La caries dental en la infancia se puede prevenir, sin embargo, continúa siendo una preocupación de salud pública importante, identificada como una de las enfermedades crónicas más prevalente de la infancia. Para disminuir los riesgos de que el niño sufra de caries de infancia temprana, se debe empezar con la educación de la madre, principalmente enfocando la importancia de una correcta higiene oral y una adecuada alimentación del niño (Tinanoff \& Risine, 2009). Se recomienda que la higiene oral del niño debe ser realizada por los padres hasta que el niño sea autónomo (8 a 10 años) (Palma \& Cahuana, 2010). La educación debe empezar en el hogar, para ello es necesario implementar programas que capaciten al padre de familia en hábitos de higiene oral saludables para que ayuden a sus hijos a llevar un control adecuado de la limpieza oral después de alimentarlos y prepararlos para que tomen conciencia de la importancia de la asistencia odontológica (Gonzáles et al., 2011).

Según Kuhn et al. (2007), los programas educativos preventivos mediante charlas a las madres utilizando la orientación verbal, afiches y proyección de slides, contribuyen a disminuir la incidencia de caries, estimulando la adopción de hábitos más saludables.

Ortiz et al. (2009) realizaron una investigación cuasi experimental con el objetivo de determinar el comportamiento de escolares, padres de familia y docentes, en el mantenimiento de la salud oral del niño. Evaluaron la condición de salud oral (caries inicial y manifiesta) y el índice de placa en niños de 6 años de edad. También determinaron el nivel de conocimientos y significados en salud oral de padres y/o madres de familia, así como de docentes. Se aplicó un modelo de capacitación con escenarios de aprendizaje participativos en docentes y padres. Después de la capacitación y análisis de los resultados obtuvieron una reducción significativa del índice de higiene oral y un aumento en el nivel de conocimientos de salud oral de padres y madres de familia.

La educación acerca de los tópicos de preven- ción, higiene oral y conocimiento de las estructuras orales tienen diferentes enfoques, sin embargo, a pesar de ser una importante labor, su éxito como programa radica en la capacidad de llegar a concientizar vía una adecuada técnica educativa a la madre a fin de obtener el máximo beneficio del programa, teniendo en cuenta, no solamente la mejora del nivel de conocimientos de salud oral de la madre, sino también el impacto en la higiene oral de los preescolares.

El objetivo de la presente investigación fue determinar la eficacia de la técnica educativa Desarrollo de Habilidades para mejorar los conocimientos de salud oral de madres y reducir el índice de higiene oral de sus hijos preescolares (grupo experimental), en comparación con la técnica educativa Expositiva (grupo control).

\section{MATERIAL Y MÉTODO}

\section{Diseño del estudio}

\section{Estudio cuasi experimental, comparativo, prospectivo y longitudinal.}

Población y muestra. La población de estudio estuvo conformada por las madres de preescolares de 3 a 5 años de edad que aceptaron voluntariamente las condiciones del estudio y por sus hijos que asistieron regularmente a la institución educativa de nivel inicial 080 adscrita a la jurisdicción de la Microrred de Salud Faucett, Callao.

La muestra estuvo constituida por 84 madres y sus hijos de esta institución educativa, de las cuales 42 constituyeron el grupo experimental que recibió la técnica educativa Desarrollo de Habilidades, y las otras 42 integraron el grupo control que recibió la técnica educativa Expositiva.

\section{Procedimientos}

La investigación se llevó a cabo en la institución educativa. Se realizó en tres etapas:

En la primera se realizó la detección del índice de higiene oral de los preescolares, utilizando el índice de higiene oral de Green-Vermillion (índice de placa) (Greene \& Vermillion, 1964). El examen oral se realizó en espacio acondicionado en el patio para utilizar la luz natural. Se sentó al niño frente al operador y se realizó la observación de la cavidad oral con ayu- 
da de un espejo oral plano $N^{\circ} 5$. Con un hisopo y sin hacer presión se dejó violeta de genciana en los siguientes dientes: 55, 51, 65 y 81 por bucal, y 75 y 85 por lingual. Luego se observó si la superficie dentaria quedó manchada y se registró de acuerdo a lo siguiente: 1 si la mancha ocupó el tercio cervical, 2 si la mancha abarcó hasta los 2/3 de la superficie dentaria, y 3 si la mancha abarcó más de $2 / 3$ de la superficie dentaria. La evaluación del índice de higiene oral se realizó al inicio del estudio, a los 30 y 60 días posteriores.

Para medir el nivel de conocimientos de las madres se elaboró un cuestionario validado de selección múltiple de 16 preguntas, con 4 respuestas cada una, siendo solamente una la correcta.

En la segunda etapa se aplicó las técnicas educativas a los grupos en las aulas. Los contenidos de las sesiones incluyeron: conocimiento de las estructuras orales, enfermedades orales, medidas preventivas y técnicas de cepillado dental.

Al grupo experimental, dividido en tres grupos de 14, se le aplicó la técnica educativa Desarrollo de Habilidades (Riquelme, 2012), que consistió en tres momentos: la exposición de contenidos teóricos, la demostración y la práctica por parte de las madres, haciendo uso de rotafolios, medios audiovisuales y material didáctico: cepillo dental y tipodón educativo para hacer las demostraciones. Todas las madres participaron en la sesión para reafirmar los conocimientos y se ejercitaron en técnica de cepillado sobre el tipodón en la primera y segunda sesión. La técnica de cepillado utilizada fue la de barrido de cervical a oclusal por vestibular y palatino cada dos dientes. En oclusal se realizó barrido horizontal. En la tercera sesión se hizo la demostración de la placa bacteriana utilizando violeta de genciana en un niño que no participó del estudio, luego se demostró la forma de eliminarla con el cepillo. La sesión duró 30 minutos. Se dictaron tres sesiones, una cada 15 días.

Al grupo control, dividido en tres grupos de 14 se le aplicó la técnica educativa expositiva. Se hizo uso de rotafolios y medios audiovisuales. La sesión duró 30 minutos. Se dictaron tres sesiones, una cada 15 días.

En la tercera etapa se evaluó la eficacia de las técnicas educativas empleadas mediante la aplicación del mismo cuestionario inicial a las madres y se realizó la evaluación final del índice de higiene oral en los preescolares hijos de las madres participantes de la misma forma que se realizó el examen inicial.
Análisis estadístico. Para la valoración de la situación pre intervención se acudió al cuestionario inicial y para la post intervención al cuestionario final. Se calcularon las medidas de frecuencia adecuadas para estimar los diferentes indicadores e índices.

Para el análisis comparativo, se midieron las variables en los momentos antes de la intervención- cuestionario $\mathrm{N}^{\circ} 1$ - y posterior a la intervención -cuestionario $\mathrm{N}^{\circ} 2$.

Para valorar las diferencias observadas en el nivel de conocimientos e índice de higiene oral en relación a la técnica educativa, se utilizó la prueba t de Student para muestras independientes y relacionadas. El nivel de significancia se fijó en 0,05, I.C. al 95\%, error del $5 \%$.

Aspectos éticos . La investigación fue aprobada por la Escuela Universitaria de Post Grado de la Universidad Nacional Federico Villarreal. Para la realización del trabajo se obtuvo el consentimiento informado de las madres y el asentimiento verbal de los niños. Los datos fueron manejados con total confidencialidad.

\section{RESULTADOS}

De los 42 preescolares evaluados pertenecientes al grupo experimental, es decir, en aquellos niños cuyas madres recibieron la técnica educativa Desarrollo de Habilidades, se encontró que el índice de higiene oral, fue disminuyendo progresivamente en cada evaluación, desde una media inicial de 2,51 hasta alcanzar una media final de 0,72. Asimismo de los 42 preescolares evaluados pertenecientes al grupo control, es decir, en aquellos niños cuyas madres recibieron la técnica educativa expositiva, se encontró que el índice de higiene oral, fue disminuyendo progresivamente de 2,51 a 1,69 (Fig. 1).

Las medias del índice de higiene oral antes de aplicar la técnica educativa en los preescolares del grupo experimental y control no mostraron diferencia significativa, es decir que se partió de situaciones similares $(p>0,05)$. Sin embargo, cuando se realizó la comparación del índice de higiene oral a los 30 días de iniciado el estudio, se encontró una diferencia altamente significativa $(p<0,001)$ entre los grupos experimental $(1,14)$ y control $(2,02)$. La misma diferencia $(p<0,001)$ se encontró también a los 60 días de evaluación, habiéndose encontrado una higiene oral de 


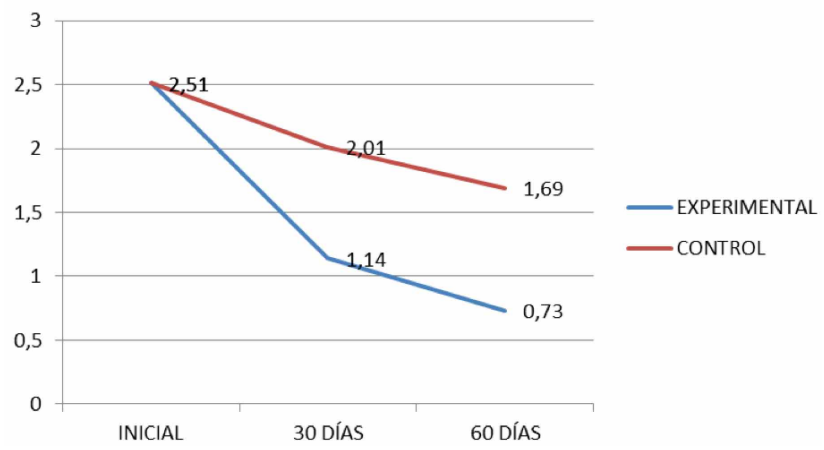

Fig. 1. Índice de higiene oral de preescolares: inicial, 30 y 60 días después en el grupo experimental y control.

0,73 en el grupo experimental y 1,69 en el grupo control. En los tres casos la comparación se realizó por medio de la prueba t de Student (Tabla I)

En relación al nivel de conocimientos de salud oral, antes de iniciar el estudio las madres del grupo experimental presentaron un nivel de conocimientos medio de 6,57 y después de la investigación aumentó a 11,88 existiendo diferencias estadísticamente significativas $(p<0,001)$. Asimismo, en el grupo control las madres presentaron un aumento en el nivel de conocimientos de salud oral alcanzando al final del estudio una media de 8,48 existiendo también diferencias significativas respecto al nivel inicial $(p<0,001)$ (Tabla II).

Al comparar las medias del nivel de conocimiento de salud oral después de aplicar las técnicas educativas, se obtuvo que la media alcanzada por las madres del grupo experimental después de la aplicación de la técnica educativa Desarrollo de Habilidades $(11,88)$ fue mayor respecto a la media alcanzada por las madres del grupo control que recibieron la técnica expositiva $(8,48)$.Esta diferencia fue altamente significativa al aplicar la prueba t de Student $(p<0,001)$ (Tabla III).

\section{DISCUSIÓN}

El presente artículo presenta la evaluación de dos técnicas educativas para mejorar los conocimientos de salud oral de madres y el impacto en la disminución del índice de higiene oral de preescolares, ya que muchas veces las madres presentan un nivel de conocimiento regular sobre salud oral, sin embargo la salud oral de sus hijos menores de cinco años es mala (Benavente et al., 2012). También se ha demostrado que en los niños existe mayor riesgo a enfermar de

Tabla I. Índice de higiene oral de los preescolares del grupo experimental y el grupo control al inicio, a los 30 y 60 días.

\begin{tabular}{lccccccc}
\hline \multirow{2}{*}{ Tiempo } & \multirow{n}{*}{} & \multicolumn{2}{c}{ Experimental } & \multicolumn{2}{c}{ Control } & \multirow{2}{*}{$\mathbf{t}$} & \multirow{2}{*}{$\mathbf{p}$} \\
\cline { 3 - 6 } & & Media & $\mathbf{D E}^{*}$ & Media & $\mathbf{D E}^{*}$ & & \\
\hline Inicio & 42 & 2,51 & 0,39 & 2,51 & 0,36 & 0,0006 & 0,995 \\
30 Días & 42 & 1,14 & 0,29 & 2,02 & 0,39 & $-11,633$ & $<0,001$ \\
60 Días & 42 & 0,73 & 0,36 & 1,69 & 0,34 & $-12,659$ & $<0,001$ \\
\hline
\end{tabular}

*DE= desviación estándar.

Tabla II. Nivel de conocimientos de salud oral de las madres antes y después de la aplicación de las técnicas educativas.

\begin{tabular}{lccccccc}
\hline Grupo & $\mathbf{n}$ & \multicolumn{2}{c}{ Antes } & \multicolumn{2}{c}{ Después } & \multirow{2}{*}{ t } & \multirow{2}{*}{$\mathbf{p}$} \\
\cline { 3 - 6 } & & Media & $\mathbf{D E}^{*}$ & Media & $\mathbf{D E}^{*}$ & & \\
\hline Experimental & 42 & 6,57 & 2,55 & 11,88 & 2,12 & $-25,445$ & $<0,001$ \\
Control & 42 & 6,55 & 2,43 & 8,48 & 2,38 & $-17,554$ & $<0,001$ \\
\hline
\end{tabular}

*DE= desviación estándar

Tabla III. Comparación del nivel de conocimientos de salud oral de las madres del grupo experimental y control después de la aplicación de las técnicas educativas.

\begin{tabular}{llccll}
\hline Grupo & $\mathbf{n}$ & Media & $\mathbf{D E}^{*}$ & $\mathbf{t}$ & $\mathbf{p}$ \\
\hline Experimental & 42 & 11,88 & 2,12 & \multirow{2}{*}{, 920} & $<0,00$ \\
Control & 42 & 8,48 & 2,38 & & \\
\hline
\end{tabular}

${ }^{*} \mathrm{DE}=$ desviación estándar. 
problemas gingivales cuando el nivel de conocimientos de las madres es bajo (Murrieta et al., 2004).

Con la aplicación de técnicas educativas a corto plazo dirigidas a madres, se puede lograr una mejora de las condiciones de salud oral de los niños preescolares, esto es posible gracias a una adecuada higiene oral (Gonzáles et al., 2006). En esta investigación, respecto a la higiene oral, ambos grupos lograron una disminución significativa de su índice de higiene, sin embargo, el grupo experimental logró una mayor disminución $(p<0,001)$, lo que indica que al aplicar la técnica educativa Desarrollo de Habilidades se obtiene mejores beneficios que con la técnica Expositiva. Estos resultados son similares a los encontrados por Hernández et al. (2004), que luego de seis meses obtuvieron una mejoría en la higiene oral después de una intervención educativa afectivoparticipativa a padres y niños.

Kuhn et al., utilizando una técnica educativa de orientación verbal haciendo uso de afiches y de diapositivas obtuvieron resultados similares de reducción del índice de higiene oral en niños. Estos resultados son corroborados por estudios previos como el de Franco et al. (2008), que mediante una intervención con estrategias educativas lograron sensibilizar a la comunidad educativa, padres, docentes y niños de 4 a 9 años, sobre la importancia de salud oral y la higiene oral, logrando una disminución importante en los índices de placa a lo largo del programa, en los primeros controles de evaluación se encontraron valores elevados de placa dental (cerca del 90\%), mientras que en el último control se evidenció una reducción importante del indicador. Resultados similares a la presente investigación donde se demostró una reducción importante del índice de higiene oral después de la aplicación de la técnica educativa Desarrollo de Habilidades a madres.

González et al. (2012), después de una intervención educativa a padres y niños entre 4 y 12 años lograron una reducción del índice de placa de los niños en un $18,3 \%$ con respecto al primer examen. Ortiz et al. también evidenciaron que hay asociación entre el antes y después de la capacitación a madres, pues encontraron que el índice de placa bacteriana final en niños de 6 años de edad tiene un nivel inferior al obtenido al inicio. Estos resultados son parecidos a los de esta investigación donde se demostró que después de la intervención educativa hay una disminución del índice de higiene oral, es decir una disminución de placa bacteriana en niños de 3 a 5 años de edad.
En el estudio se ha podido demostrar que la aplicación de la técnica educativa Desarrollo de Habilidades mejora significativamente el nivel de conocimiento de salud oral de las madres de preescolares, probablemente influenciadas por la sesión educativa que consistió no sólo en la transmisión de conocimientos teóricos sino en actividades demostrativas y prácticas en tipodones didácticos. Estos resultados son similares a los obtenidos por González et al. (2006, 2012), que después de la segunda encuesta a los padres encontraron un aumento en el conocimiento de todas las áreas de salud oral que se ve reflejado en varios aspectos como lo relacionado con la visita al odontólogo, la cual no solo se hace para solucionar problemas odontológicos sino también para lograr una buena salud oral, resaltando la importancia de cuidar los dientes deciduos.

Hernández et al. evaluaron el nivel de información de educación para la salud sobre caries dental de padres y/o tutores al concluir una intervención educativa afectiva participativa, observando que alcanzaron una evaluación buena el $81 \%$ de los padres y sólo un $3 \%$ obtuvo la calificación de mal. Asimismo, hay coincidencia con los reportes de Ortiz et al., que comparando el antes y después de la capacitación con estrategias participativas a los padres y madres de familia, el nivel de los conceptos en salud oral mejoraron de una manera estadísticamente significativa. Resultados similares a los obtenidos en la presente investigación. Por otro lado, se espera que estudios como este sensibilicen a las autoridades educativas, de salud y padres de familia en temas de prevención de las enfermedades orales.

Se debe considerar que esta investigación trabajó solo con preescolares provenientes de una institución educativa estatal, por lo que se recomienda realizar estudios similares en niños de instituciones particulares o que no asisten a ninguna institución educativa.

Además, es necesario estudios adicionales para corroborar los resultados obtenidos en esta investigación, así como evaluar las técnicas educativas dirigidas a madres de familia y su relación con la incidencia de enfermedades orales, especialmente de gingivitis y caries de infancia temprana en preescolares.

Se concluye que la técnica Desarrollo de Habilidades demostró ser más eficaz en el aumento del nivel de conocimientos de salud oral de madres y en la disminución del índice de higiene oral de preescolares. 
MUÑOZ-CABRERA, W. E. \& MATTOS-VELA, M. A. Educational technique to improve the knowledge of oral health of mothers and reduce the index of oral hygiene of preschoolers. Int. J. Odontostomat., 9(2):321-327, 2015.

ABSTRACT: The objective of this study was to determine the efficiency of the educational technique Development of Skills to improve the knowledge of oral health of mothers and reduce the index of oral hygiene in pre-school. A quasiexperimental study was conducted. A validated questionnaire on knowledge of oral health was given to 84 mothers before and after the application of the educational technologies Development of Skills and Explanatory (control group). The mothers were assigned to the experimental and the control groups in random form. Was evaluated oral hygiene of their pre-school children from the start, to 30 days and to 60 days, using the simplified of Greene-Vermillion index. Initially the average level of knowledge of oral health went from 6.57 for the experimental group and 6.55 for the group control. After the intervention the mean of the experimental group was 11.88 and for the control group 8.48, with a statistically significant difference $(p<0.001)$. As regards to oral hygiene, average index at baseline, 30 and 60 days in the experimental group were $2.51,1.14$ and 0.73 while in the control group were $2.51,2.02$ and 1.69 respectively. There were statistically significant differences between groups at 30 and 60 days $(p<0.001)$. The technique Development of Skills demonstrated to be more effective in the increase of the level of knowledge of oral health of mothers and in the decrease of the index of oral hygiene in pre-schoolers.

KEY WORDS: knowledge, mothers, oral health, oral hygiene index.

\section{REFERENCIAS BIBLIOGRÁFICAS}

American Academy of Pediatric Dentistry. Policy on Early Childhood Caries (ECC): Classifications, Consequences and Preventive Strategies. Pediatr Dent. Reference Manual, 33(6):47-49, 2011-2012.

Benavente, L. A.; Chein, S. A.; Campodónico, C. H.; Alva, E. P.; Ventocilla, M. S.; Castro, A.; Huapaya, O. et al. Nivel de conocimientos en salud bucal de las madres y su relación con el estado de salud bucal del niño menor de cinco años de edad. Odontol. Sanmarquina, 15(1):14-8, 2012.

Castañeda, M.; Maita, L. \& Romero, M. Caries dental e higiene bucal en pacientes atendidos en la Clínica del Niño, Facultad de Odontología de la Universidad Nacional Mayor de San Marcos. Odontol. Sanmarquina, 12(1):18-21, 2009.

Elías, M. Efectos de la Aplicación del Programa Piloto de Atención Odontopediátrica desde el Nacimiento hasta los 36 meses de vida en el Hospital Central de la FAP para la Disminución de Caries Dental 1989-2001. Tesis de Maestría. Lima, Universidad Inca Garcilaso, 2003.

Franco, A.; Martínez, C.; Álvarez, M.; Coutin, I.; Larrea, I. \& Otero, A. Los niños tienen la palabra: Nuestras bocas sanas para una vida sana. Instituto de Ciencias de la Salud - CES. Medellín, 20(2):9-16, 2008.

González, C.; Navarro, J. \& López, P. Cambio de indicadores de placa dentobacteriana, gingivitis y caries dental en niños entre 2 y 5 años de edad a partir de una intervención educativa dirigida a madres de preescolar. Medellín 2003-2005. CES Odontología, 19(1):9-17, 2006.
González, F.; Sierra, C. \& Morales, I. Conocimientos, actitudes y prácticas en salud bucal de padres y cuidadores en hogares infantiles, Colombia. Salud Pública Mex., 53:247-57, 2011.

González, S.; Giraldo, M. C.; Varela, J.; Peña, E. M.; Giraldo, J. P. \& Orozco, J. J. Saluteca oral con enfoque familiar: hacia una nueva orientación en educación de la salud bucal. Rev. Fac. Odontol. Univ Antioq., 23(2):306-20, 2012.

Granville-García, A. F.; Ferreira, J. M.; Barbosa, A. M.; Vieira, I.; Siqueira, M. J. \& Menezes, V. A. Cárie, gengivite e higiene bucal em pré-escolares. Rev. Gaúcha Odontol, Porto Alegre, 58(4):469-473, 2010.

Greene, J. C. \& Vermillion, J. R. The simplified oral hygiene index. J. Am. Dent. Assoc., 68:7-13, 1964.

Hernández, A. M.; Espeso, N.; Reyes, F. \& Landrián, C. Promoción de salud para la prevención de caries en niños de 5 a 12 años. $A M C, 13(6): 0-0,2009$.

Kuhn, E.; Wambier, D. S. Incidência de Lesões de Cárie em Bebês Após 15 Meses de um Programa Educativo-Preventivo. Pesqui. Bras. Odontopediatria Clín. Integr., 7(1):75-81, 2007.

Medina-Solís, C. E.; Maupomé, G.; Pelcastre-Villafuerte, B.; Ávila-Burgos, L.; Vallejos-Sánchez, A. A. \& CasanovaRosado, A. J. Desigualdades socioeconómicas en salud bucal: caries dental en niños de seis a 12 años de edad. Rev. Invest. Clín., 58(4):296-304, 2006.

Murrieta, J.; Juárez, L.; Linares, C. \& Zurita, V. Prevalencia de gingivitis en un grupo de escolares y su relación con 
el grado de higiene oral y el nivel de conocimientos sobre salud bucal demostrado por sus madres. Bol. Méd. Hosp. Infant. Méx., 61(1):44-54, 2004.

Ministerio de Salud del Perú (MINSA). Prevalencia nacional de caries dental, fluorosis del esmalte y urgencia de tratamiento en escolares de 6 a 8, 10, 12 y 15 años, Perú. 2001-2002. Lima-Perú, Ministerio de Salud del Perú, 2005.

Ortiz, L.; Gutiérrez, M.; Moromi, H.; Medina, K. \& Villavicencio, J. Identificación del comportamiento de escolares y padres de familia respecto al mantenimiento de la salud bucal. Odontol. Sanmarquina, 12(1):13-7, 2009.

Palma, C. \& Cahuana, A. Pautas para la Salud Bucal en los primeros años de vida. Odontol Pediatr., 9(2):179-89, 2010.

Petersen, P. E. The World Oral Health 2003. Continuous improvement of oral health in the 21 st century the approach of the WHO Global Oral Health Programme. Ginebra, World Health Organization, 2003.

Riquelme, M. Metodología de educación para la salud. Rev. Pediatr. Aten. Primaria, Supl. (21):77-82, 2012.

Tinanoff, N. \& Risine, S. Update on Early Chilhood Caries since the Surgeon General's Report. Acad. Pediatr., 9(6):396-403, 2009.

Zaror, S. C.; Pineda, T. P. \& Orellana, C. J. J. Prevalencia de caries temprana de la infancia y sus factores asociados en niños chilenos de 2 y 4 años. Int. J. Odontostomat., 5(2):171-7, 2011.
Dirección para Correspondencia:

Dr. Welser Muñoz-Cabrera

Dirección Regional de Salud

Callao

PERÚ

Email:welserm17@gmail.com

Received: 18-11-2014

Accepted: 27-04-2015 